\title{
Improving care for patients with idiopathic pulmonary fibrosis (IPF) in the UK: a round table discussion
}

\author{
David R Thickett, ${ }^{1}$ Clare Kendall, ${ }^{2}$ Lisa G Spencer, ${ }^{3}$ Nick Screaton, ${ }^{4}$ \\ William A Wallace, ${ }^{5}$ Hilary Pinnock, ${ }^{6}$ Julia Bott, ${ }^{7}$ Lucy Pigram, ${ }^{8}$ Sarah Watson, ${ }^{9}$ \\ Ann B Millar ${ }^{10}$
}

\begin{abstract}
- Additional material is published online. To view please visit the journal (http:/l dx.doi.org/10.1136/thoraxjnl2014-206284)

For numbered affiliations see end of article.

Correspondence to Professor David Thickett, Centre for Translational Inflammation and Fibrosis Research, University of Birmingham, Edgbaston, B15 2TH, UK

d.thickett@bham.ac.uk
\end{abstract}

Received 10 September 2014 Accepted 21 September 2014 Published Online First 22 October 2014

CrossMark

To cite: Thickett $D R$

Kendall C, Spencer LG, et al.

Thorax 2014;69:

$1136-1140$

\section{ABSTRACT}

This article is the result of a round table discussion held at BMA House. Its purpose is to outline the challenges facing both patients and doctors in managing idiopathic pulmonary fibrosis. A number of recommendations for service development and research have been identified.

\section{BACKGROUND TO THE ROUND TABLE DISCUSSION}

In June 2013, the National Institute of Health and Care Excellence (NICE) published its first clinical guideline on the diagnosis and management of idiopathic pulmonary fibrosis (IPF), ${ }^{1}$ but a narrow scope and a limited evidence base, in terms of published placebo-controlled trials and cost-effectiveness data, constrained the recommendations that could be made. With the emergence of novel and effective therapy for patients with IPF, it is clear that IPF care will evolve significantly over the next few years.

Recognising the need for prompt diagnosis and management of patients with IPF, and the limitations of the existing NICE guideline, Thorax undertook an electronic poll that suggests that currently there is significant variation in management of patients with IPF (see online supplement), and therefore convened an advisory round-table discussion to highlight problem areas and agree additional recommendations to improve care. This paper is the result of those discussions.

\section{Implications of epidemiology and disease course}

IPF is a chronic, progressive fibrotic interstitial lung disease (ILD) of unknown origin. ${ }^{2}$ The incidence of IPF and registered deaths from this cause in the UK continues to rise in the 21 st century with more than 5000 new cases diagnosed each year in the UK. ${ }^{3}$ Due to its poor prognosis, this means that at present, perhaps, 15000 patients have a diagnosis of IPF with 5000 deaths per year.

The prevalence of IPF is around 15-25 per 100000 and increases with age. The condition is rare in people younger than 45 years of age, and the median age of presentation is 70 years of age. This means that the average hospital with a catchment of 500000 will have 40-45 new cases per year, and a typical general practitioner's (GP) practice of 10000 patients will see $\sim 1$ new case every year. ${ }^{3}$ Most patients with IPF experience breathlessness, which may initially be only on exertion, and cough, with or without sputum, is also common. Over time, there is a progressive decline in lung function, reduced quality of life and, ultimately, death.

Prognosis is difficult to estimate at the time of diagnosis. When deterioration occurs, it can be rapid. Median survival for IPF from diagnosis in the UK is approximately 3 years, and only about $20 \%$ of people will survive for more than 5 years. ${ }^{1}$ The likelihood for rapid deterioration and the detrimental impact of that on quality of life means that early diagnosis and appropriate management is vital. $^{4}$

\section{PRIMARY CARE-WHEN TO SUSPECT ILD}

A patient with IPF will commonly present to their GP with breathlessness or cough, both of which are very common symptoms. About $1.1 \%$ GP consultations are related to breathlessness, and cough is the cardinal symptom of $4.6 \%$ of consultations. ${ }^{5}$ By contrast, IPF is a relatively rare condition, with which GPs will have little personal experience. At most, a full-time GP can only expect to identify one new case of IPF every 7 years. ${ }^{3}$ The challenge for a GP is, therefore, to identify IPF from among the most common causes of breathlessness.

In such rare conditions, symptom-based algorithms which help GPs manage common symptoms like breathlessness and cough will be more practical than disease-based pathways. Red flags to alert GPs to circumstances where patients need investigation for sinister or rare conditions could enable appropriate referrals to breathlessness services. ${ }^{6}$

From the perspective of identifying IPF, crackles with breathlessness could be one such red flag, highlighting the need for a chest X-ray which could lead to earlier recognition of interstitial changes and specialist referral. Although often a chest X-ray report is inconclusive in general practice, it is a gateway to specialist referral even if the initial referral route, cardiology versus respiratory, for example, is incorrect.

A restrictive spirometry measurement (widely undertaken in UK primary care) is another potential red flag, though both performance and interpretation need to be quality assured. ${ }^{7}$ The gold standard investigation for IPF is high-resolution CT (HRCT), to which few GPs have direct access.

Primary care has a very important role in supporting the patient and their family at all stages of the condition, particularly in recognising the need for, and providing of, palliative care. Because of IPF's rarity, the role of new treatments is likely to 
be unfamiliar to most GPs, and the management of end-of-life care needs, especially the intractable breathlessness, may be perceived as challenging.

\section{Learning/action points}

- IPF is a rare condition, whereas, the initial presenting symptoms of breathlessness and cough are common in general practice. GPs need symptom-based algorithms to help them identify which patients warrant further investigation by X-ray and spirometry, and subsequent referral to a respiratory specialist.

- GPs need more information on the likely disease course in patients with IPF and the role of treatment modalities, and will often require the support of specialist nursing and palliative care services, as supportive care is increased with the approach of end of life.

\section{EARLY ACCURATE DIAGNOSIS IS KEY FOR NEW THERAPIES}

Clinical experience suggests that patients with IPF often present quite late in the disease course having had symptoms for many months/years prior to diagnosis. They have often first been (mis) diagnosed with other more common respiratory diseases like asthma or COPD before an IPF diagnosis is reached. Thus, for some, the potential of starting therapy early to prevent lung function decline is lost.

New drug therapies are now available that can slow disease progression and that have an impact on survival, so earlier diagnosis of IPF is of increasing importance. Furthermore, there is some evidence that delayed diagnosis of IPF is associated with increased costs in terms of investigations performed, and delayed referral can result in lower survival rates independent of disease severity or associated prognostic factors. ${ }^{8}{ }^{9}$ It is clear, therefore, that to optimally manage patients with IPF we need to be able to diagnose accurately and early. We need to raise the diagnostic suspicion of IPF in primary and secondary care and encourage early referral for assessment to ILD specialists.

\section{Learning/Action Points}

- Improvements to the diagnostic pathway are needed because patients perceive delays in obtaining a timely and accurate diagnosis.

\section{ROLE OF, AND ACCESS TO, SPECIALIST IPF CARE}

The process of accessing specialist IPF care should become easier and clearer than it currently is in some areas, as the ongoing ILD commissioning process is completed (see below). Regions will be expected to form networks with recognised referral pathways in place agreed between referring hospitals. The specialist ILD centres will have knowledge of, and access to, all new drugs for the treatment of IPF and other ILDs, and where possible, offer patients access to clinical trials in this field.

Patients will continue to present to their local respiratory consultants who will still take the history, examine the patient, order appropriate tests and manage the patient on a day-to-day basis, but diagnostic and management decisions will be taken with the support of the regional ILD multidisciplinary teams (MDTs) as needed or/as agreed by local referral pathways. Pathways should broadly follow the standards of care as set out in the ILD commissioning document drafted by the respiratory Clinical Reference Group (CRG).

Travelling long distances to specialised centres can become increasingly burdensome for patients with a deteriorating respiratory condition, so wherever possible, shared care between local and specialist centre should be provided. Recognition of the need of those in the more palliative phases of their disease requiring local care is important. Clear communication between centres is going to be increasingly important and valuable in managing patients across several sites.

\section{Learning/action points}

- Ensuring that patients with suspected IPF are referred to the ILD MDT will help ensure equitable access to specialist radiological and pathological diagnoses.

- A balance needs to be struck between centralisation and access to practical community care, and virtual MDTs may have a role.

\section{NURTURING THE ILD/IPF SPECIALISTS OF THE FUTURE}

Currently, there is no 'ILD accredited pathway' for registrar training in respiratory medicine, so few applicants for specialist ILD jobs can gain the desired depth of experience prior to starting as a consultant. Courses in ILD are available, but only one masters (Sci) level ILD module in Liverpool is currently known to exist run via Respiratory Education UK.

Training attachments in ILD need to be considered at specialist centres to address this. Thought also needs to be given to the training requirements and expertise of radiology and pathology staff to produce excellent ILD MDT members for the future.

\section{Learning/action points}

- Consideration should be given to the levels of training and expertise that MDT members require, and how that will be delivered in the future.

\section{RADIOLOGY AND PATHOLOGY CONTRIBUTION TO DIAGNOSIS OF IPF}

The gold standard imaging test for suspected IPF is HRCT because a high-quality report from a radiologist with an interest in ILD can identify IPF and rule out many other conditions.

Patients with suspected IPF should be sent for HRCT as early as possible and a report requested from a chest radiologist with experience of IPF. Where this expertise is unavailable at the hospital where the imaging takes place, the image can be sent electronically to a chest radiologist at a specialist centre for primary reporting or to gain a second opinion via a hub-and-spoke, or network model.

Where radiological findings suggest that non-specific changes are inconclusive, patients may be sent for pathology. Patients tend to be sent for lung biopsy if HRCT shows atypical lung architecture changes and is inconclusive. If the lung architecture is atypical on HRCT scan, it will often appear atypical on biopsy as well. Some patients may have had prebiopsy treatment which will reflect on the findings, because the sample taken will be partially treated ILD which looks different to untreated ILD. As a result, interpretation of the pathological changes is frequently not straightforward leading to well-recognised problems with interobserver variation both between generalist and specialist pathologists ${ }^{10}$ and between specialists. ${ }^{11}$

A surgical lung biopsy is the only specimen that will allow specific identification of a usual interstitial pneumonia (UIP) pattern. There has been great debate about where the biopsies should be taken from, but the latest evidence suggests taking them from two lobes and avoiding areas of end-stage fibrosis. ${ }^{12}$

It is important to realise that a pathological report cannot make a diagnosis of IPF. The report will describe the pattern of lung remodelling present which, in most patients with IPF, will be UIP. ${ }^{13}$ This information on the pattern of remodelling then has to be put together with the clinical features by the ILD MDT to make a diagnosis of IPF, as similar patterns of 
remodelling may be seen in patients with connective tissue disorders or drug reactions.

The role of bronchoalveolar lavage in IPF is highly controversial, and there is no evidence that it can provide a specific diagnosis, although it may provide evidence supporting a clinical/ radiological diagnosis. ${ }^{14}$ Similarly, transbronchial and imageguided percutaneous lung biopsies have no role in the diagnosis of IPF, but may be useful in identifying other potential diagnoses. ${ }^{15}$

\section{Learning/action points}

- A clear definition is required of what are the most appropriate radiology and pathological investigations for IPF, when they should be conducted, how they should be reported and their limitations.

- A clear definition is required of the skills requirements for people who report these investigations (specialism, training, volume of cases).

- Training pathways are required to attract young radiologists and pathologists and provide a structure for their development.

- Consideration needs to be given into how pathology and radiology fits into the ILD MDT structure on a regional level to enhance the quality of diagnostic information provided.

- Pathology and radiology on its own does not provide a diagnosis.

\section{NON-PHARMACOLOGICAL THERAPIES FOR IPF Pulmonary rehabilitation}

There is a limited robust evidence on the benefits of pulmonary rehabilitation (PR) in IPF because inadequate high-quality studies have been conducted and this needs to be addressed, although there was sufficient evidence to allow NICE recommendation that PR should be offered at diagnosis (NICE 2013). ${ }^{1}$

The thorax survey suggests significant variation in the availability and use of PR in IPF (see online supplement). PR should be funded for patients with IPF in all regions/areas.

All individual components of PR should be offered to patients, even if it is at a late stage. There is evidence that breathing retraining can help control exertional and panic-related tachypynoea. ${ }^{16}$ If early referral for PR is missed or was not accepted, a fatigue and breathlessness programme specifically for palliative patients rather than a standard programme may be more appropriate.

\section{Occupational therapy}

Patients with IPF will also benefit from occupational therapy (OT), ideally from a respiratory specialist, to provide advice on energy conservation in activities of daily living (how to bathe, shave, dress, etc), stress management and appropriate home equipment or adaptations. Again, due to the potential for rapid deterioration, referral to OT and provision of aids needs to be timely.

\section{Ambulatory oxygen}

People with IPF frequently demonstrate a fall in oxygen saturation during exercise. In such people, ambulatory oxygen is often provided to improve exercise capacity, enhance mobility and enable activities of daily living in order to improve quality of life. ${ }^{1}$ Currently, there are no randomised controlled trials to demonstrate that ambulatory oxygen therapy is effective in achieving these aims in patients with IPF, thus, assessment on an individual basis is necessary. ${ }^{17}$ The thorax survey suggested significant support for ambulatory oxygen therapy, but variation when it was offered to patients. A 6 min walk or shuttle walk test will provide the most accurate indication of exercise-induced desaturation and the required level of oxygen supplementation.

\section{Long-term oxygen therapy}

Resting hypoxaemia and the need for long-term oxygen therapy (LTOT) is often indicative of end-stage disease. There are no trials that demonstrate that LTOT influences outcome in IPF. However, it has been shown that nocturnal hypoxaemia is common and may impact on quality of life. ${ }^{18}$ LTOT is often used, therefore, but should be titrated appropriately to prevent potentially harmful hyperoxia.

\section{Learning/action points}

- PR should be made more widely available to patients with ILD.

- Patients diagnosed with ILD should be fast tracked into PR.

- Rapid access into OT and physiotherapy is also required because of the potential for rapid deterioration.

- High levels of ambulatory O2 may be required on exertion, but LTOT needs to be prescribed carefully to avoid potentially harmful hyperoxia.

\section{THE ROLE OF THE ILD SPECIALIST NURSE}

The ILD nurse specialist provides a central link between the patient, their carer/s and all healthcare services, including the GP, hospital consultant and palliative care services. Services available to patients differ from area to area, and there are insufficient specialist nurses for the current volume of work.

An important consideration when creating specialist centres is that patients are likely to be at considerable distance from the expertise of specialist staff. In recognition of the importance of the ILD specialist nurse role for patient care, the group recommended that if services are to be centralised, more ILD specialist nurse posts will need to be funded. This will facilitate the provision of a responsive specialist service alongside GPs and palliative care services close to the patient in addition to their work within the regional centres.

IPF is a disease that patients struggle to understand, which can heighten their anxiety. From the very start, patients need to be given clear and consistent information on its course, management, and care, in line with the British Lung Foundation's IPF Patient Charter. ${ }^{19}$

\section{Learning/action points}

- More patient support groups are required.

- A register of services, such as Macmillan nurses, respiratory nurses and community matrons available in each area would be helpful to assist ILD specialist nurses with interdisciplinary liaison.

\section{ACCESS AND PROVISION OF PALLIATIVE CARE}

All patients with IPF are likely to have palliative care needs at some point. It can be challenging for professionals to judge the optimum timing of referral but it is important to get it right because there is a growing body of evidence showing unmet palliative needs in the ILD patient group. ${ }^{20}$

There are known markers of progressive disease, which should prompt referral to palliative care services including FVC decline of $10 \%$ or more, gas transfer (TLCO) $<30 \%$, increasing hypoxia, increasing oxygen requirements for same level of activity, progression of disease on HRCT scan, an exacerbation requiring admission to hospital in the last 3 months, significant 
decline in personal functional status requiring assistance for personal activities of daily living, and being confined to a chair for $>50 \%$ of the waking day. As not all staff delivering palliative care will have much experience in caring for patients with IPF, it is important that all professionals involved are made aware of the disease trajectory.

Patients with more complex needs should have input from specialist palliative care services either in the acute hospital or in the community. As well as symptom management, palliative care should encompass full holistic care by also addressing psychological, social, practical, financial and information needs. The group agreed that the aim of advance care planning in ILD should be about ensuring that the patient receives appropriate care towards the end of life.

Although NICE guideline on IPF says that discussion about end of life should begin at diagnosis, this is controversial. However, it is important to start explaining to the patient about the disease course and management so that they know what to expect. $^{21}$ The exact content of the discussion should be sensitively tailored to the individual, and is a process which will evolve over time.

\section{CURRENT AND EMERGING DRUG THERAPY}

NICE has recently recommended pirfenidone as a treatment option for patients with IPF who have a FVC of between 50\% and $80 \%$ predicted, and are assessed as having IPF by a specialist ILD MDT. Centres for prescription have been identified through the pharmacological services and NHS England. Only specialist dedicated centres can obtain reimbursement from NHS England for pirfenidone.

Pirfenidone (Esbriet, Intermune) has been shown to reduce the progression of the disease in terms of decline of FVC, progression-free survival and IPF-related mortality. ${ }^{22}$ The NICE criteria restriction of an upper limit of FVC of $80 \%$ is significantly restricting its prescription even in those with biopsyproven UIP, whose estimates vary throughout the country, but between $25 \%$ and $50 \%$ of cases discussed at ILD MDT have FVC over $80 \%$ predicted (D Thickett, unpublished observations).

There are many more drug molecules in development for IPF, targeting multiple pathways including the integrin $\alpha v \beta 6$ and transforming growth factor $\beta$ signalling, anti-IL-13 and anti-LOXL2 antibody (see ref. 23 for a recent review). A multifunctional oral tyrosine kinase inhibitor is the only drug, apart from pirfenidone, to have successfully completed phase III testing. It is not licensed for use in any country. The two phase III INPULSIS trials demonstrated efficacy in terms of declining lung function, but mixed results in terms of quality-of-life changes and exacerbations. ${ }^{24}$ There is no current information about effects of the tyrosine kinase inhibitor upon mortality.

We are therefore at last able to offer active drugs to IPF patients with the prospect of slowing disease decline. However, the current drugs do not stop or reverse decline, and have significant side effects that limits tolerability in a significant proportion of patients in the real world outside clinical trials. The drugs have also only been tested in mild to moderate IPF.

The pathophysiology of IPF is complex involving fibroproliferation, ${ }^{2}$ epithelial cell apoptosis, ${ }^{25}$ epithelial-mesenchymal transition, ${ }^{26}$ neo-angiogenesis, ${ }^{27}$ and intra-alveolar coagulopathy. As with most effective lung disease treatments, it is unrealistic for us to expect a single magic bullet to be universally effective. It is expected that ultimately patients will be treated with combination therapy, so comparative trials of potential new drugs alone and in combination will be needed. Currently, there are only a few centres in the UK which regularly participate in ILD studies for new drugs and biomarkers.

\section{Learning/action points}

- Patients should be offered the opportunity to be entered into these trials particularly at the more severe end of the disease spectrum.

- Pharmaceutical companies and the IPF clinical community should work towards assessment of combination therapy perhaps with factorial trials design to facilitate patient recruitment.

\section{COMMISSIONING OF SPECIALIST SERVICES BY NHS ENGLAND IS NEEDED URGENTLY}

In April 2013, as part of large reorganisation of the NHS, the newly formed NHS England through CRGs have developed more than 175 new service specifications, setting out the requirements from providers of specialised services in terms of the quality of care and treatment offered. Three of these specialist services are in respiratory medicine including services for people with ILD, including IPF.

Specialised services currently account for around $10 \%$ of the total National Health Service (NHS) budget. NHS England's responsibilities are discharged through 27 area teams, 10 of which have responsibility for commissioning specialised services. NHS England has undertaken a considerable piece of work aimed at better understanding the extent to which providers of specialised services are currently meeting key elements of the standards, this work is ongoing. Along with a small number of other specifications, the implementation of the specifications for complex home ventilation and ILD was paused for 2013/2014 because more refinement of clinical coding was required to appropriately determine specialised activity. The pause has also enabled the specifications to be reviewed by the respiratory CRG to ensure that they take into account the new NICE guideline on IPF.

In line with the implementation of all specialised commissioning specifications, NHS England is undertaking a compliance programme assessing all current providers of ILD services against a set of key requirements from the specification. Area teams are now working with providers to commission the services and once arrangements have been agreed then the list of ILD centres will be made public.

Until specialised ILD services have been commissioned, trusts which have ILD expertise and have historically provided advice and support to neighbouring trusts are being paid to continue with these activities. However, while some sort of service is being provided, it may not meet the quality dictated by the service specifications, and local services and patients are unlikely to be clear on how the network arrangements work.

\section{Learning/action points}

- We need to get specialist centres commissioned and networks in place.

- We need to avoid the disparity of service across the UK that may occur as the result of disparity between the speeds of progress of Area Teams in commissioning respiratory services.

- Ease of patient access should be considered by area teams when deciding where to locate specialist centres and the possibility of outreach centres or sub-hubs where centres cover particularly large geographical areas or areas with poor transport. 
- Much can be learned from experience in pulmonary hypertension, where there are a small number of specialist centres surrounded by effective networks.

- Optimising the use of technological solutions to ensure timely communication and effective transmission of data while reducing the need for travel.

\section{CONCLUSIONS}

With the publication of NICE guidance on IPF and NICE approval of pirfenidone in IPF, clear markers of quality of patient care have been established. The delays in specialist commissioning for patients with ILD have meant that patients have unacceptable differing access to specialist care and treatment in different regions of the UK. The round-table discussion has outlined further areas for research and patient pathway improvement. All who attended felt these were promising times for patients and those tasked with helping them cope with their disease.

\author{
Author affiliations \\ ${ }^{1}$ Centre for Translational Inflammation and Fibrosis Research, University of \\ Birmingham, Edgbaston, UK \\ ${ }^{2}$ Palliative Medicine, North Bristol NHS Trust, Bristol, UK \\ ${ }^{3}$ Aintree Chest Centre, Aintree Hospital, Liverpool, UK \\ ${ }^{4}$ Department of Radiology, Papworth Hospital, Cambridge, UK \\ ${ }^{5}$ Department of Pathology, Edinburgh, UK \\ ${ }^{6}$ Allergy and Respiratory Research Group, Edinburgh, UK \\ ${ }^{7}$ Respiratory Care Team, Surrey PCT, London, UK \\ ${ }^{8}$ Respiratory Medicine, Royal Brompton Hospital, London, UK \\ ${ }^{9} \mathrm{NHS}$ England, Edgbaston, UK \\ ${ }^{10}$ Lung Research Group, University of Bristol, Bristol, UK
}

Contributors A round-table discussion hosted and organised by Thorax, and summary edited by DRT. Chair: ABM. Respiratory Medicine: DRT and LGS.

Radiology: NS. Pathology: WAW. GP: HP. Physiotherapist: JB. Nurse: LP. Policy: SW. Palliative Care: CK.

Funding This initiative is sponsored by Boehringer Ingelheim through the provision of an unrestricted educational grant. Boehringer Ingelheim has had no influence over the content other than a review for medical accuracy.

Competing interests $A B M$, president of the British Thoracic Society, member of NICE guidelines group for IPF, South-West representative for the respiratory clinical reference group and involvement in clinical trials of therapies for IPF (InterMune, Boehringer-Ingelheim, Gilead), no personal remuneration. LP part-time employment as a telephone support nurse for Partizan healthcare on IPF. Assisted to go to conferences by InterMune and Boehringer, and received speaker fees by both companies. LGS has received travel sponsorship to attend respiratory conferences from Intermune and Boehringer Ingelheim, payment for advisory board work from InterMune (twice) and Boehringer Ingelheim (once with one future event pending) (f1000 or less per event). WAW accepted an invitation from InterMune to participate in sponsored Scottish IPF Update Meeting 27th August 2014 for no financial reward. HP, none in relation to IPF. JB has received a fee from Boehringer Ingelheim for contributing to a BLF DVD for patients on exercise in the home. DRT has received travel sponsorship to attend respiratory conferences from Intermune and Boehringer Ingelheim, and was a trialist on the IMPULSIS trial. ${ }^{24}$

Provenance and peer review Not commissioned; internally peer reviewed. ${ }^{6}$

\section{REFERENCES}

1 NICE. The diagnosis and management of suspected idiopathic pulmonary fibrosis. NICE clinical guideline 163, 2013.

2 Richter AG, McKeown S, Rathinam S, et al. Soluble endostatin is a novel inhibitor of epithelial repair in idiopathic pulmonary fibrosis. Thorax 2009;64:156-61.
3 Navaratnam V, Fleming KM, West J, et al. The rising incidence of idiopathic pulmonary fibrosis in the U.K. Thorax 2011;66:462-7.

4 Cottin V, Richeldi L. Neglected evidence in idiopathic pulmonary fibrosis and the importance of early diagnosis and treatment. Eur Respir Rev 2014;23:106-10.

5 Okkes IM, Oskam SK, Lamberts $H$. The probability of specific diagnoses for patients presenting with common symptoms to Dutch family physicians. J Fam Pract 2002:51:31-6.

6 IMPRESS. Breathlessness IMPRESS Tips. http://www.networks.nhs.uk/nhs-networks/ impress-improving-and-integrating-respiratory

7 Levy ML, Quanjer PH, Booker R, et al. Diagnostic spirometry in primary care: Proposed standards for general practice compliant with American Thoracic Society and European Respiratory Society recommendations: a General Practice Airways Group (GPIAG)1 document, in association with the Association for Respiratory Technology \& Physiology (ARTP)2 and Education for Health3 $1 \mathrm{http}: / /$ www.gpiag. org2 http://www.artp.org 3 http://www.educationforhealth.org.uk. Prim Care Respir J 2009;18:130-47.

8 Ley B, Collard HR, King TE Jr. Clinical course and prediction of survival in idiopathic pulmonary fibrosis. Am J Respir Crit Care Med 2011;183:431-40.

9 Lamas DJ, Kawut SM, Bagiella E, et al. Delayed access and survival in idiopathic pulmonary fibrosis: a cohort study. Am I Respir Crit Care Med 2011;184:842-7.

10 Lettieri CJ, Veerappan GR, Parker JM, et al. Discordance between general and pulmonary pathologists in the diagnosis of interstitial lung disease. Respir Med 2005;99:1425-30.

11 Nicholson AG, Addis BJ, Bharucha $\mathrm{H}$, et al. Inter-observer variation between pathologists in diffuse parenchymal lung disease. Thorax 2004; 59:500-5.

12 Raghu G. Idiopathic pulmonary fibrosis: guidelines for diagnosis and clinical management have advanced from consensus-based in 2000 to evidence-based in 2011. Eur Respir / 2011;37:743-6.

13 Raghu G, Collard HR, Egan JJ, et al. An official ATS/ERS/JRS/ALAT statement: idiopathic pulmonary fibrosis: evidence-based guidelines for diagnosis and management. Am J Respir Crit Care Med 2011;183:788-824.

14 Meyer KC, Raghu G, Baughman RP, et al. An official American Thoracic Society clinical practice guideline: the clinical utility of bronchoalveolar lavage cellular analysis in interstitial lung disease. Am J Respir Crit Care Med 2012;185:1004-14.

15 Bradley B, Branley HM, Egan JJ, et al. Interstitial lung disease guideline: the British Thoracic Society in collaboration with the Thoracic Society of Australia and New Zealand and the Irish Thoracic Society. Thorax 2008;63(Suppl 5):v1-58.

16 Bott J, Blumenthal S, Buxton M, et al. Guidelines for the physiotherapy management of the adult, medical, spontaneously breathing patient. Thorax 2009;64(Suppl 1):i1-51.

17 Nishiyama O, Miyajima H, Fukai $\mathrm{Y}$, et al. Effect of ambulatory oxygen on exertional dyspnea in IPF patients without resting hypoxemia. Respir Med 2013;107:1241-6.

18 Egan JJ. Follow-up and nonpharmacological management of the idiopathic pulmonary fibrosis patient. Eur Respir Rev 2011;20:114-17.

19 Foundation. BL. IPF Patient Charter.

20 Temel JS, Greer JA, Muzikansky A, et al. Early palliative care for patients with metastatic non-small-cell lung cancer. N Engl J Med 2010;363:733-42.

21 Parker S, Clayton JM, Hancock K, et al. A systematic review of prognostic/end-of-life communication with adults in the advanced stages of a life-limiting illness: patient/ caregiver preferences for the content, style, and timing of information. J Pain Symptom Manage 2007;34:81-93.

22 King TE Jr, Bradford WZ, Castro-Bernardini S, et al. A phase 3 trial of pirfenidone in patients with idiopathic pulmonary fibrosis. $N$ Engl I Med 2014;370:2083-92.

23 Woodcock HV, Maher TM. The treatment of idiopathic pulmonary fibrosis. F1000prime Rep 2014;6:16.

24 Richeldi L, du Bois RM, Raghu G, et al. Efficacy and safety of nintedanib in idiopathic pulmonary fibrosis. N Engl J Med 2014;370:2071-82.

25 Kuwano K. Involvement of epithelial cell apoptosis in interstitial lung diseases. Intern Med 2008;47:345-53.

26 Bartis D, Mise N, Mahida RY, et al. Epithelial-mesenchymal transition in lung development and disease: does it exist and is it important? Thorax 2014;69:760-5.

27 Dancer RC, Wood AM, Thickett DR. Metalloproteinases in idiopathic pulmonary fibrosis. Eur Respir / 2011;38:1461-7. 